\title{
Getting to grips with fraud
}

\section{Dealing with scientific fraud is likely to be an unpleasant process for accused and accuser alike. The} only solution lies in well conceived methods of procedure.

UNTIL recently there would seem to have been little need for discussion of the dangers of the fraudulent reporting of data to the scientific enterprise. There was always the odd historical case of fraud and every generation seemed to bring forth at least a handful of people who were determined to identify finally the perpetrators of the Piltdown Man hoax. But that was a pastime conducted in a similar obsessive spirit to the hunt for the identity of the "dark lady" of Shakespeare's sonnets and seemed to have little relevance to the daily practice of the scientific profession.

In retrospect, 1980 proved a turning point with allegations of falsification of data made in four major cases. The pace of discovery of new cases of fraud, malpractice and plagiarism has not slackened subsequently. Since 1980 , such allegations have been made publicly in at least twenty-five cases and cleared up privately in a further seven cases.

These are the figures, not necessarily comprehensive, that obtained at a workshop on scientific fraud and misconduct sponsored jointly by the American Association for the Advancement of Science and the American Bar Association last week. The figures are not by themselves particularly worrying. Even though more than a hundred papers tainted by fraud may have been produced, that number is still insignificant compared to the world output of scientific literature. But there remain several issues to worry about.

How are scientific frauds detected? Conventional wisdom has it that science is self-correcting: experiments are repeatable and a fraudulent or incorrect result will inevitably be detected in a later repetition. But in much of the biological sciences, where a great measure of skill and experience is needed to master new techniques, things are not so simple. And in the medical sciences, where human subjects are involved, experimental opportunities may have to be taken as they arise, and cannot be totally controlled or perfectly repeated. To say these things does not call into question the basic principle that experiments are repeatable. But it does mean that the first thought of someone who fails to replicate a result is not likely to be that the result is fraudulent. A more charitable explanation is likely to be sought in minor differences in experimental conditions.

For these reasons it appears that frauds have rather rarely been discovered through the replication of experiments by totally independent groups. The discovery of fraud is more usually a personal affair: a co-worker's suspicions are aroused, and then confirmed by events extraneous to the published record - for example, that a reagent essential to the experiment had not been ordered at that time, or even that the number of Petri dishes used in an experiment was greater than the number the laboratory had in stock.

The personal nature of the discovery of fraud inevitably makes it a painful business. It is not like having been a chance witness at a bank robbery. No one is going to emerge as a hero from an investigation which is likely to blacken not just the name of the individual scientist but also his or her institute and the scientific enterprise itself. But that should not mean that the person who exposes a fraud is penalized for his or her courage.

Unfortunately, the experiences of "whistle-blowers", personally related at the workshop, makes clear that the price of exposing fraud is high. Action by institutions, which clearly saw their own reputations at stake, was often taken grudgingly and after years of delay. The motives of the accuser were frequently questioned: did jealousy or personal animosity play a part?

There seems no escaping the conclusion that for both accuser and accused to have full protection of their good reputations, which is ultimately all that a scientist has to assure continued employment, universities need effective processes for dealing with allegations of fraud and dispensing justice rapidly. Some universities - mainly those who have been through the protracted trauma of a fraud case - have already made a start but their experiences show that it is not easy. The skills for investigating fraud, different from those necessary for research, are hard to obtain. And universities have genuine conflicts of interest between dispensing justice and protecting their reputation that may make systematic policies and procedures unpalatable.

There is little hope that universities transfer their responsibility to investigate fraud to other bodies (except when the misuse of public funds makes it necessary to involve the granting agencies). Scientific societies may help to set guidelines of proper practice but they are not in a position to deal with individual cases. Nor can a great deal of help be expected from scientific journals. Although journals may choose to publish letters that point out inconsistencies in, or failures to replicate, previously published work, they would find themselves in a difficult legal position if they began to make accusations about their own authors.

Could journals do more to ensure that fraudulent work never appears in print? "Why ever was the paper accepted" is a natural enough reaction when a case of fraud is uncovered. But evidence of fraud is not necessarily to be found in the the published account. And while conscientiousness can be expected of referees, the total scepticism needed to suspect that an author's every statement may be false, surely cannot. Even scepticism may be insufficient for fraudulent work is often correct in its conclusions. That need cause no surprise. People who commit fraud are only very rarely stupid enough to falsify scientific breakthroughs. More characteristic is a pedestrian piece of work with a wholly expected result. But that that is so forces a hard question: How different is fraud from shoddy practice?

If the timid manufacture of data to conform to already probable hypotheses is the most common form of fraud is it one end of a spectrum of shoddy practice that includes the selection of data, the filling in of inadequate or control data through phentom experiments, and the use of guest co-authors to add credibility to mediocre work? The more comforting view is that frauds are committed by a few highly deviant individuals whose activities have nothing to do with the normal scientist. Between these two hypotheses - that fraud represents either the tip of an iceberg or a few bad apples - there are not data to make a judgement, although practising scientists clearly each have their own view. But a corollorary of the first hypothesis is that there are rewards for the mediocre work that no one will ever read carefully. That leads to a much larger question: has the scientific enterprise come to stress quantity to such an extent that quality has begun to suffer? 